\title{
Sistemas de pago a prestadores de servicios de salud en países de América Latina y de la OCDE
}

\author{
Begoña Álvarez, ${ }^{1}$ Laura Pellisé ${ }^{1}$ \\ y Félix Lobo ${ }^{1}$
}

RESUMEN Además de ser uno de los componentes más importantes de las relaciones contractuales entre los agentes de los sistemas de salud, el sistema de pago a prestadores es relevante para algunos de los principales criterios de valoración de un sistema de salud, como la eficiencia y la calidad. En este artículo tratamos de proporcionar un marco analítico sencillo para comprender la na turaleza de los distintos sistemas de pago, que ilustramos con una revisión de las fórmulas de pago utilizadas en dos grupos de países: 10 de la Organización de Cooperación y Desarrollo Económico (OCDE) y cuatro de América Latina cuyas experiencias consideramos representati vas. Primero presentamos un modelo básico para caracterizar las diferentes formas de pago, ba sado en dos dimensiones: la unidad de pago y la distribución de riesgos financieros entre el que vende y el que compra. Cada sistema de pago tiene ventajas e inconvenientes que deben eva luarse en función de los objetivos que se pretenda alcanzar. En un extremo tenemos la remune ración fija, que representa el pago prospectivo más puro, sin ajustes, como el pago capitativo, en el que los prestadores soportan todo el riesgo financiero derivado de la variabilidad de costos. En el otro extremo se sitúa el pago retrospectivo o por acto, con el que el riesgo incurrido por los prestadores es nulo y es el financiador quien soporta todo el riesgo derivado de la variabilidad de costos. Como suele ocurrir, los extremos no parecen óptimos y la cuestión consiste en esco ger un sistema de remuneración intermedio. Para ello, es necesario seleccionar, por una parte, la unidad de pago óptima según los objetivos del financiador y, por otra, una distribución de riesgos que atribuya al prestador los riesgos derivados del mayor o menor grado de eficiencia que consiga en la prestación de los servicios.

El sistema de pago a prestadores es uno de los componentes más impor-

\footnotetext{
Seminario de Estudios Sociales de la Salud y los Medicamentos, Instituto Flores de Lemus, Universidad Carlos III, Madrid, España. Toda la correspondencia debe ser enviada a Begoña Álvarez a la siguiente dirección postal: Instituto Flores de Lemus, Universidad Carlos III de Madrid, C/Madrid, 12628903 Getafe, Madrid, España. Correo electrónico: flobo@eco.uc3m.es
}

tantes de las relaciones contractuales entre los agentes de los sistemas de salud y, en realidad, uno de sus signos definidores. Constituye, además, una cuestión relevante para algunos de los principales criterios de valoración de un sistema de salud, como la eficiencia y la calidad. Los sistemas de pago son especialmente importantes para la efi- ciencia del sistema, fundamentalmente porque las decisiones sobre gasto $\mathrm{y}$ nivel de producción dependen, en última instancia, de los profesionales sanitarios y ocurre que las fórmulas de pago y los incentivos que estas generan son capaces de influir sobre ellos. También tienen relevancia desde el punto de vista de la calidad, porque pueden 
afectar a la forma de ejercicio de la medicina, al trato al paciente, etc.

En torno a los sistemas de pago se han generado, y se siguen generando, un sinfín de discusiones y coexisten múltiples opciones que bien podrían entenderse como un reflejo de la falta de superioridad de unos sobre otros. De hecho, no existe ningún sistema de pago "natural" al que deban acogerse los distintos sistemas de salud. Cada sistema de pago tiene ventajas e inconvenientes que deben evaluarse en función de los objetivos que se pretenda alcanzar.

Esta diversidad se puede explicar, en primer lugar, por la coexistencia de multitud de agentes que intervienen en el sector. Entre estos agentes se establecen, implícita o explícitamente, relaciones que suponen una contraprestación económica. Básicamente podemos distinguir a los prestadores de asistencia (médicos, otro personal asistencial, hospitales, centros asistenciales, etc.), a los individuos (usuarios potenciales), tanto si están asegurados como si no lo están, y a las aseguradoras, públicas, como la Seguridad Social, o privadas, como las organizaciones de mantenimiento de la salud (health maintenance organizations: HMO), etc. Estos agentes se relacionan entre sí mediante flujos financieros y reales que generan una multitud de sistemas de reembolso.

En segundo lugar, la diversidad de las fórmulas de pago también está relacionada con la heterogeneidad del producto ofrecido por los agentes sanitarios y con la asimetría de la información entre las partes contratantes. Por ejemplo, la contratación de servicios hospitalarios por parte de una aseguradora puede implicar un reembolso por presupuesto global, por ingreso, por día de estancia u otros.

En este artículo tratamos de proporcionar al lector un marco analítico sencillo para comprender la naturaleza de los distintos sistemas de pago que hoy están vigentes en la mayoría de los países. El estudio se ilustra con una revisión de las fórmulas de pago utilizadas en dos grupos de países: uno de 10 países de la Organización de Coopera- ción y Desarrollo Económico (OCDE) y otro de cuatro países de América Latina cuyas experiencias consideramos representativas.

En la siguiente sección presentamos un modelo básico para caracterizar las diferentes formas de pago. A continuación analizamos los criterios de selección de sistemas de pago óptimos y posteriormente presentamos un análisis sintético de las fórmulas de pago utilizadas en los países estudiados. El artículo finaliza con una serie de conclusiones generales.

\section{UN MODELO SENCILLO DE ANÁLISIS DE LOS SISTEMAS DE PAGO}

El marco conceptual que presentamos está basado en el trabajo de Pellisé (1). Por sistemas de pago (o fórmulas de remuneración) entendemos la forma en que se organiza la compensación económica, normalmente monetaria, que recibe un prestador sanitario por la prestación de sus servicios a un paciente o grupo de pacientes. Estas contraprestaciones se pueden clasificar en una tipología relativamente corta de categorías que centrarán nuestra exposición (por ejemplo, salario, pago por acto, pago por ingreso hospitalario, etc.). El prestador sanitario puede ser un profesional individual o una entidad colectiva, organizada como empresa privada o como institución pública (por ejemplo, un hospital o una clínica de atención primaria).

Es necesario distinguir aquí entre el problema de la financiación de los servicios de salud y los sistemas o fórmulas de pago o remuneración a los prestadores. El primero es básicamente un problema macroeconómico que alude a las fuentes originarias de financiación del sistema de salud y a los criterios y mecanismos de asignación de recursos a los distintos subsistemas. En cambio, los sistemas de pago plantean una cuestión básicamente microeconómica, que afecta al comportamiento de los agentes económicos a nivel individual y configura sus in- centivos económicos y el riesgo al que se ven sometidos en el ejercicio de su actividad.

La literatura especializada considera que los sistemas de pago tienen en común la característica de que todos pueden describirse con dos dimensiones: la unidad de pago y la distribución de riesgos financieros entre el que vende y el que compra. Veremos cada una de estas dimensiones por separado.

\section{La primera dimensión: la unidad de pago}

Cada sistema de pago a prestadores utiliza una unidad de pago particular que se aproxima, en mayor o menor grado, a una medida de los insumos utilizados (por ejemplo, tiempo de trabajo de un médico, por el cual se le paga un salario), del producto (número de personas inscritas cuya asistencia global queda comprometida en el capitativo) o de los productos intermedios (ingreso, estancia $\mathrm{u}$ otros).

Las unidades de pago se distinguen por el grado de agregación de los servicios asistenciales contratados. Puede considerarse que la unidad menos global o más desagregada es el pago por servicio y las unidades más agregadas son, sucesivamente, los pagos por un proceso asistencial, por una estancia hospitalaria, por un ingreso y por la asistencia a un enfermo a lo largo de un determinado período de tiempo (por ejemplo, pago capitativo, o cualquier forma de aseguramiento sanitario integral).

Este criterio de ordenación es sumamente transcendente, ya que determina el tipo de incentivo que el financiador emite al prestador, al determinar qué productos y qué insumos entran en juego en el concepto de eficiencia que se está incentivando. Por ejemplo, si el pago es capitativo, la señal que emitimos es que el prestador tendrá ingresos adicionales si aumenta el número de individuos que tiene adscritos, mientras que sus ingresos no variarán aunque aumente la intensidad de la atención por indivi- 
duo adscrito. Si este pago se realiza como contrapartida por una atención integral de la salud, como ocurre con las HMO, con los médicos de familia bajo el sistema británico reformado (General Practitioner Fundholders) o con las entidades mutuales en España, entonces los incentivos en pro de la eficiencia afectarán a todos los servicios asistenciales y podrá conseguirse la satisfacción de los adscritos (suponiendo posibilidad de elección y economías de escala) con un nivel mínimo de costes gracias a la utilización racional de los servicios.

\section{La segunda dimensión: los riesgos financieros}

Una vez establecida la unidad de pago, existe todavía un amplio abanico de posibilidades para definir la fórmula de remuneración. Por ejemplo, una vez seleccionado el ingreso hospitalario como unidad de pago, aún nos queda por elegir entre muchas posibilidades, como establecer un solo precio, cualquiera que sea el origen y motivo del ingreso, o diseñar una tarifa basada en grupos de diagnóstico relacionado (GDR) o en función del número de productos hospitalarios diferenciados (tipos de estancia) que reconozca el financiador.

Cada forma de remuneración conlleva riesgos financieros distintos. Para valorarlos, debemos tener en cuenta dos aspectos fundamentales: la distribución desigual de los costos hospitalarios y la distribución, por tramos o intervalos, de la remuneración.

Tomemos el ingreso hospitalario como unidad de pago. Ahora cabe preguntarse si, más o menos, todos los ingresos cuestan lo mismo. $\mathrm{Si}$ fuese así, el 50\% de los ingresos hospitalarios más baratos estarían asociados al $50 \%$ de los costos del hospital. Sin embargo, en la mayoría de los hospitales se da una distribución desigual, es decir, hay un alto porcentaje de ingresos relativamente baratos $y$ un porcentaje menor de ingresos que generan costos muy elevados. Por ejemplo, podría ocurrir que los ingre- sos hospitalarios más caros representasen solo $20 \%$ del total de ingresos, pero absorbiesen $80 \%$ de los costos del hospital.

En cuanto a la distribución por tramos de las remuneraciones, pongamos por caso que existe un único asegurador, público, y que debe decidir cómo pagará a los hospitales por cada ingreso. En un extremo, podría pagar un tanto fijo y único, con lo que implícitamente estaría reconociendo que todos los ingresos son iguales. Alternativamente, el financiador podría establecer una tarifa simple y diferenciar tres tipos de ingresos distintos, cada uno pagado a un precio diferente, o establecer una tarifa compleja y distinguir casi 500 intervalos de precio correspondientes a 500 productos diferentes, como el sistema de pago a hospitales en Estados Unidos de América (EUA), basado en GDR. Finalmente, en el otro extremo, podría pagar una cantidad distinta por cada ingreso, presuponiendo que cada estancia en cada hospital es un producto diferente. Así, podrían existir tantos precios como ingresos producidos.

Tomando en cuenta los razonamientos anteriores, puede deducirse que, para una dada unidad de pago, cuanto más nos acercamos al caso de la remuneración consistente en un tanto fijo (es decir, cuanto menor es el número de los tramos o intervalos en cuyo espectro puede oscilar la remuneración $\mathrm{y}$, por tanto, más amplios son estos) más se arriesga el prestador si los costes varían. $\mathrm{O}$, más sintéticamente, cuanto mayor sea la variabilidad de costos para un precio dado, mayor riesgo financiero se transfiere del financiador hacia el prestador.

\section{Caracterización de los tipos fundamentales de sistemas de pago}

A partir del modelo que acabamos de exponer, esto es, considerando la unidad de pago de que se trata y la distribución del riesgo entre financiador y prestador/oferente de servicios de salud, vamos a caracterizar los dis- tintos sistemas de pago vigentes en la mayoría de países.

En el cuadro 1 se incluyen tres formas de pago a médicos (pago por servicio o por acto, pago por salario y pago por capitación) y tres formas de remuneración a hospitales (pago por día o por estancia, pago por ingreso y pago por presupuesto).

Para cada una de ellas, además de describir su unidad de pago y la distribución de riesgo que conlleva, analizamos los siguientes aspectos: incentivo económico fundamental, características y efectos previsibles, relación con la eficiencia, relación con la calidad y posibilidades de acción pública (cuadros 2 y 3$)^{2}$

Como caso extremo se considera una remuneración fija, o sea, con un solo intervalo. Es el pago prospectivo más puro, o sin ajustes, como el pago capitativo. Los prestadores soportan todo el riesgo financiero derivado de la variabilidad de costos para una tarifa prefijada y una unidad de pago. En el otro extremo, tenemos el caso del pago retrospectivo o pago por acto (fee-for-service en inglés). El riesgo incurrido por los prestadores es nulo: sea cual sea el costo de la unidad producida, la remuneración lo cubrirá y el financiador será quien soporte todo el riesgo derivado de la variabilidad de los costos.

$\mathrm{Ni}$ un extremo ni otro parecen óptimos. La distribución de riesgos entre prestadores y financiador es muy importante, al igual que lo es la elección de la unidad de pago que se quiere remunerar. La cuestión es, entonces, saber escoger un sistema de pago eligiéndolo de entre una gama amplísima de posibilidades, como veremos a continuación.

\footnotetext{
2 Es preciso subrayar que nuestra exposición se refiere únicamente a los incentivos económicos y a la dirección hacia la que moverían la conducta de los profesionales sanitarios si no existiera ninguna otra motivación. Naturalmente esto es una simplificación, pues no tiene en cuenta motivaciones distintas de las económicas, que pueden ser tan importantes o más que estas, como el altruismo o la reputación.
} 


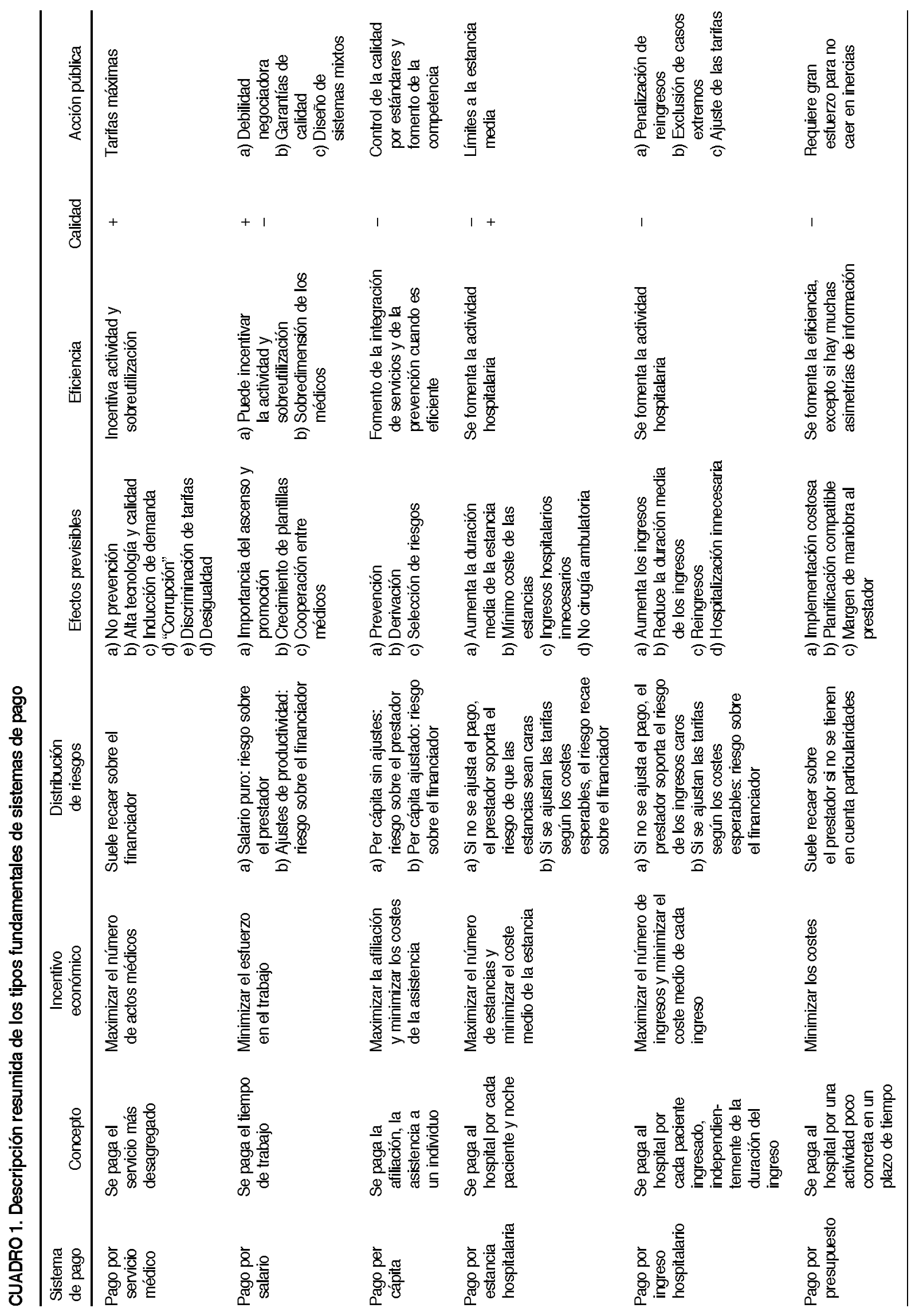




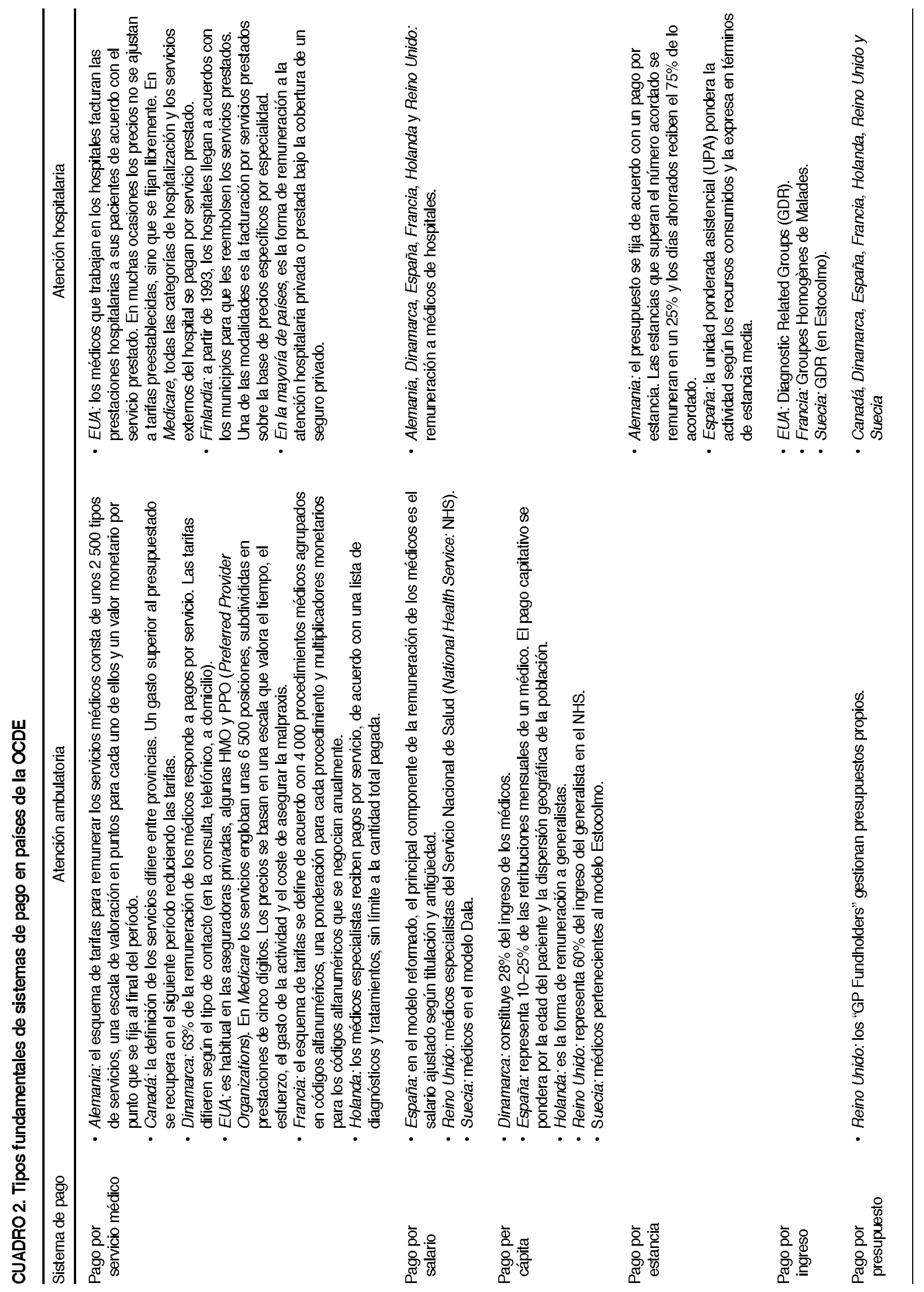




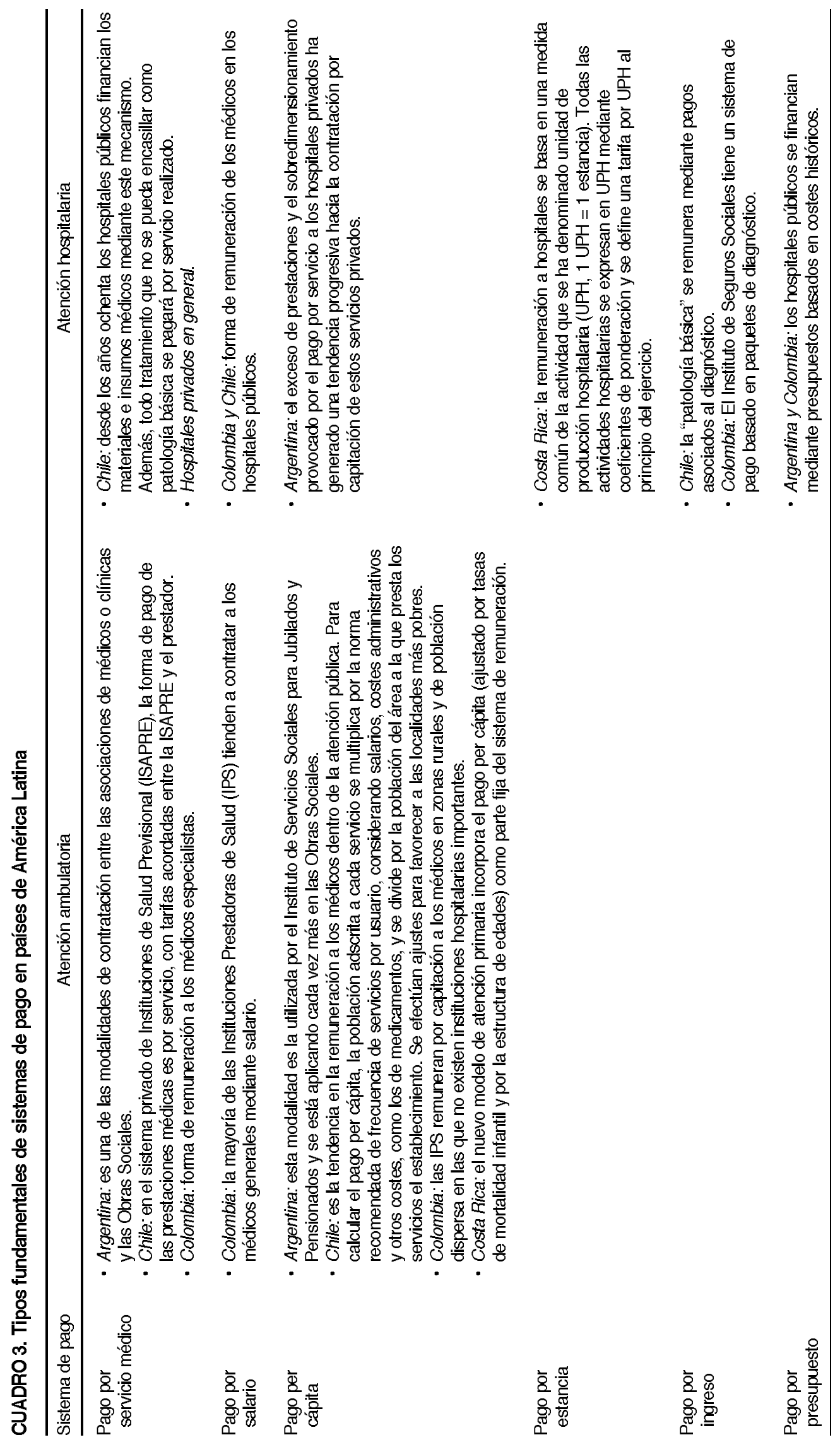




\section{LA ELECCIÓN DEL SISTEMA ÓPTIMO DE PAGO: UN BALANCE ENTRE EFICIENCIA Y RIESGOS FINANCIEROS}

Todos los sistemas de pago pueden definirse en función de dos vectores. El primero corresponde a los incentivos (dependientes de servicios que se remuneran dentro de la unidad de pago definida). El segundo consiste en la distribución de riesgos. A medida que se refinan las tarifas según las características de la asistencia, el riesgo financiero se traslada desde el prestador hacia el financiador. De la combinación de los dos vectores descritos resulta una gama de posibles sistemas de remuneración. El diseño de un sistema óptimo de financiación pasa necesariamente por escoger un valor para cada vector: unidad de pago y riesgo. La elección de la unidad de pago debe realizarse entre las fórmulas que se mencionaron en el epígrafe anterior. En principio, no existe una unidad de pago válida para todo tiempo y lugar. La elección depende, entre otras cosas, de los objetivos del financiador. Sin embargo, existe un cierto consenso acerca de las virtudes de cada una de ellas, como se expuso en el cuadro 1.

En cuanto a la elección óptima de la distribución de riesgos, los analistas de este aspecto resaltan el abismo existente entre la teoría y la práctica. Teóricamente, los costos unitarios de un prestador de servicios de salud pueden variar por diversas razones:

a. Por una buena o mala gestión. Se trata, por tanto, de costos "controlables" por el gestor, que podrán calificarse de "injustificables" si son sistemáticamente muy elevados.

b. Por la naturaleza del caso médico, cuya variabilidad da lugar a variaciones "incontrolables" de los costos, "justificables" y sistemáticas.

c. Por razones aleatorias cuya predicción o anticipación resulta muy costosa y que serían también "justificables".

Desde un punto de vista normativo, sería deseable una distribución de ries-

\section{La reforma de las obras sociales en Argentina}

\author{
Secretaría de Salud Pública \\ de la Municipalidad de Rosario (Argentina) ${ }^{1}$
}

\begin{abstract}
Durante los años noventa en Argentina se presenciaron profundos cambios que pueden valorarse de diferentes maneras, según el énfasis que se coloque en los logros alcanzados o en los problemas que no han sido resueltos, como las manifestaciones de exclusión, violencia y desintegración social. Pocos argentinos desconocen, sin embargo, que en esos años el país comenzó a superar algunos problemas que afectaron a su desarrollo económico y social a lo largo de cuatro décadas: inestabilidad política, crecimiento económico insatisfactorio, inserción inadecuada en el mercado mundial, déficit fiscal permanente, conflictos sociales no resueltos, poca legitimidad de las instituciones democráticas y serias limitaciones en la capacidad de regulación de los aparatos del Estado.

No obstante, los logros alcanzados - la democracia y la recuperación económica, las perspectivas favorables para la inversión extranjera y la buena evaluación de las políticas implantadas por parte de las grandes instituciones- no son suficientes para saldar la gravísima deuda social que el país generó en los años de inestabilidad y deterioro de su capacidad productiva. Por el contrario, esta deuda se incrementó a posteriori como consecuencia de la concentración económica derivada del proceso de cambio y hoy en día se traduce en pobreza y marginalidad en las zonas urbanas, desnutrición infantil y deserción escolar, desempleo, ruptura de los vínculos de integración social, y una sociedad que gravita hacia la dualidad.

El proceso de reforma de la atención médica se ubica en este contexto caracterizado por la expansión simultánea de la riqueza y la marginalidad. Sin embargo, la transformación de las instituciones de salud no responde solamente a las consecuencias de esta nueva situación, sino que debe tomar a su cargo el agotamiento del anterior modelo de organización de la atención médica, los conflictos no resueltos en el sistema político, y la escasa capacidad de los organismos estatales que controlan y regulan el sector de la salud para imponer las nuevas reglas del juego.
\end{abstract}

${ }^{1}$ Correo electrónico: mfein@rosario.gov.ar 
gos que atribuyera al prestador los riesgos derivados del mayor o menor grado de eficiencia que alcance en el suministro de los servicios. Deberían diseñarse tarifas con intervalos que cubrieran las variaciones de costos justificables.

Una desacertada distribución de riesgos entre financiador y prestador puede tener grandes inconvenientes. En efecto, no castigar suficientemente variaciones injustificables de los costos supone destruir incentivos a favor de la eficiencia y estimula la manipulación de riesgos. Por el contrario, no reembolsar suficientemente ciertos costos justificables sistemáticos puede originar problemas importantes en términos de selección de riesgos.

La manipulación de riesgos consiste en intentar clasificar la unidad de pago atendida en la categoría (o intervalo) que suponga mayores ingresos marginales. ${ }^{3}$ Este efecto se produce cuanto mayor es el número de intervalos y cuanto más manipulables sean las variables que definen la pertenencia a cada uno de ellos.

Por otra parte, la selección de riesgos consiste en intentar captar $a d$ hoc afiliados relativamente sanos o afecciones relativamente baratas y en esquivar afiliados previsiblemente enfermos o afecciones relativamente caras (2). Las consecuencias negativas para los pacientes y las aseguradoras pueden ser importantes en términos de costos de transacción, de información y de reputación a largo plazo. El problema es que se desconoce dónde está la frontera que separa las variaciones injustificables, debidas a ineficiencias, de las justificables. Estas están fuertemente relacionadas con la heterogeneidad del producto sanitario y sus causas quedan generalmente fuera del control del prestador (por ejemplo, la localización geográfica, que puede afectar al precio de los insumos y al tipo de paciente atendido). Estas variaciones pueden provocar problemas importantes de injusticia en la distribución de los recursos (por ejemplo, entre hospitales con presupuesto global no sensible a

\footnotetext{
3 En Estados Unidos de América esta práctica, en el caso de los GRD, se denomina "DRG creep".
}

\section{El diagnóstico que encuadró la reforma}

La primera medida tomada por el Ministerio de Economía, que asumió el liderazgo del proceso de reforma, se encaminó a hacer más transparente el manejo de los recursos destinados al sistema - hasta ese momento recaudados de forma independiente por cada Obra Social (OS) - y el número de sus beneficiarios, para así facilitar el control de la evasión. Con ese propósito en 1991 se puso en marcha una estrategia de centralización de los aportes y contribuciones a la seguridad social. El decreto de desregulación de la economía (n. ${ }^{\circ}$ 2.284/91) creó el Sistema Único de Seguridad Social y estableció la Contribución Única de la Seguridad Social (CUSS) con miras a unificar los aportes y contribuciones al sistema previsional con aquellos destinados a financiar las prestaciones de la atención médica. Un porcentaje de ellos se deriva posteriormente a las respectivas OS. ${ }^{2}$

En 1993 la política de reforma avanzó en los dos aspectos en los que se centraba el diagnóstico crítico de las OS. El primero se refería a la obligación impuesta a cada trabajador de canalizar sus contribuciones del seguro obligatorio a la OS del respectivo sindicato. Los impulsores de la reforma pensaban que ese mecanismo creaba una población "cautiva", con lo cual no habría incentivos para mejorar la calidad y cantidad de los servicios ni para administrar más eficientemente los recursos disponibles. Según ese diagnóstico, la generación de cierto nivel de competencia en la captación de beneficiarios entre las distintas OS podría, por una parte, estimular una mayor eficiencia en la gestión de la cobertura, y por otra, producir una concentración de los beneficiarios del sistema en grandes instituciones capaces de generar un reparto de riesgos adecuado. De ese modo se esperaba atenuar las grandes diferencias en el número de beneficiarios y en la disponibilidad de recursos que recurrentemente se aducían como causa de la disparidad de los beneficios otorgados a la población a cargo de cada obra social.

El segundo componente crítico giró en torno a la creciente proporción de beneficiarios de las OS que debía acudir a los servicios del subsistema público para recibir atención, fenómeno que tiene su origen en la insuficiente cobertura brindada por muchas OS que imponen al beneficiario copagos relativamente onerosos

\footnotetext{
${ }^{2}$ Hasta esta década, las OS funcionaban como cajas de seguro contra enfermedad, de afiliación obligatoria para los trabajadores en relación de dependencia y administradas por el sindicato correspondiente a cada una de las grandes ramas de actividad. Cuando se puso en marcha la reforma, existían más de 300 OS en el régimen nacional, con muy diferente capacidad para garantizar la cobertura de sus respectivos beneficiarios, dadas las notables diferencias en el tipo de miembros y en los recursos financieros a su disposición. La regulación del sistema está en manos de la Administración Nacional del Seguro de Salud (ANSSAL), agencia formalmente dependiente del Estado pero estrechamente asociada con el poder sindical.
} 
en el momento de recibir atención. Como resultado, la población de menores ingresos recurre al hospital público, que atiende a la totalidad de la población de forma gratuita, identificándose como indigente. Esta circunstancia dio origen a la idea de poner en práctica mecanismos de cobro a las OS por parte de los hospitales y centros de salud, a fin de generar nuevos recursos que alivien su crónica condición de desfinanciamiento y deterioro.

Conforme a ese diagnóstico, el decreto n. ${ }^{\circ}$ 9/93 sancionó los fundamentos de la reforma, estableciendo en primer lugar la libre elección de OS por los beneficiarios del sistema. Los titulares del beneficio podrán optar entre las diferentes OS y cambiar de opción una vez por año. También se produjeron adelantos en la reforma de las relaciones entre las OS y los prestadores de servicios, ${ }^{3}$ estableciéndose la libertad de contratación entre financiadores y prestadores, sin sujeción a honorarios o tarifas prefijadas y posibilitándose de esa manera la competencia de precios entre los prestadores de atención médica.

Por último, se estableció que las OS deberán pagar los servicios que sus beneficiarios soliciten a los hospitales públicos. Esta disposición se completó con el decreto n. $^{\circ} 578$ de ese mismo año, que reguló el funcionamiento del "hospital de autogestión" dentro del subsistema público.

El decreto n. ${ }^{\circ}$ 9/93 procuró transformar radicalmente las relaciones de poder que constituían el fundamento del sistema de servicios previamente vigente, cuyo agotamiento era reconocido por todos los actores involucrados. Dichas relaciones definían las principales áreas de intervención y estaban respaldadas por los aciertos de la política económica y la decidida aprobación del Fondo Monetario y el Banco Mundial. Sin embargo, tuvieron un acatamiento poco uniforme: la aplicación de la libre elección por parte de los beneficiarios de las OS recién se concretó en 1997; la organización de hospitales de autogestión en el subsistema público tuvo una aceptación limitada y solo se aplicó a título de ensayo en algunas instituciones, sin producir cambios significativos en los mecanismos de financiación de ese subsistema; la libre contratación entre OS y prestadores privados, por el contrario, llegó para sancionar una situación que, de hecho, había supuesto los cambios más importantes en el sistema de financiación y provisión de servicios. Más adelante se presenta una descripción de ese proceso. Ahora interesa analizar el conflicto político e ideológico desatado por la decisión de desregular el funcionamiento de las OS.

\footnotetext{
${ }^{3}$ Hasta ese momento, los contratos entre las OS y el sector privado estaban bajo el control de las grandes confederaciones de profesionales y sanatorios privados, que en condiciones de oligopolio de oferta, acordaban con las OS la atención de los beneficiarios bajo modalidades de libre elección del prestador por el paciente, libertad de prescripción por parte del profesional y pago por acto médico.
}

ellas), problemas de eficiencia (prestadores con riesgos bajos reembolsados por un precio unitario muy superior al coste marginal del mercado competitivo) y reacciones orientadas hacia la selección de riesgos.

Todas estas dificultades explican el desarrollo de una multitud de propuestas de sistemas de clasificación (de pacientes, de enfermedades, de procesos, de ingresos hospitalarios, etc.) que intentan, entre otras cosas, distribuir óptimamente el riesgo entre financiador $y$ prestador. Por ejemplo, en cuanto a unidades de pago capitativo, existen múltiples propuestas. Igualmente se han desarrollado bastantes clasificaciones de estancias hospitalarias. Sin embargo, ninguno de estos sistemas de clasificación ha dado muestras de ser óptimo. No dejan de ocasionar distribuciones de riesgos inadecuadas, con problemas de manipulación y selección de riesgos.

Para resolver el dilema entre los pagos prospectivos, que plantean dificultades en la distribución de riesgos, y los retrospectivos, que fallan al proporcionar incentivos al derroche y la ineficiencia, los sistemas mixtos han creado esperanzas. Sin embargo, no resuelven el problema de la selección de la unidad de pago. Estas formas de remuneración se basan en una mezcla de pagos prospectivos (con un número finito de intervalos) y retrospectivos (número infinito de intervalos). Se pretende así encontrar una solución intermedia entre la excesiva variabilidad de costos dentro de cada intervalo en el caso de los pagos prospectivos, que redundan en un excesivo riesgo para los prestadores, pero poniendo coto a la ineficiencia y a la ausencia de variabilidad dentro de los intervalos que muestran los pagos retrospectivos, que eliminan el riesgo para el prestador y anulan todo incentivo a favor de la eficiencia.

\section{FORMAS DE PAGO A PRESTADORES EN PAÍSES DE LA OCDE Y DE AMÉRICA LATINA}

En esta sección tratamos de analizar las características de los principales 
sistemas de pago a prestadores de servicios de salud existentes en 10 países de la OCDE (Alemania, Canadá, Dinamarca, España, Estados Unidos, Finlandia, Francia, Holanda, Reino Unido y Suecia) y en cuatro países latinoamericanos (Argentina, Chile, Colombia y Costa Rica). En este estudio nos hemos centrado en las formas de pago a la atención ambulatoria y hospitalaria. Para una descripción más detallada de las mismas, puede consultarse Álvarez et al. (3).

A diferencia de los países de la OCDE seleccionados, la información sobre las formas de pago vigentes en los sistemas de salud de América Latina es bastante limitada. La ausencia de estudios sobre los sistemas de pago en la mayoría de estos países no puede dejar de interpretarse como un síntoma del escaso protagonismo que las formas de pago han recibido tradicionalmente en el diseño de los sistemas sanitarios. En cualquier caso, creemos que las experiencias del grupo de países seleccionados son suficientemente ilustrativas y representan de forma adecuada la orientación de los cambios que se están produciendo en otros países de América Latina.

Los documentos de referencia en el caso de los países de la OCDE han sido los propios informes de esta organización $(4,5)$, complementados de manera importante con publicaciones más recientes $(6,7)$. En cuanto a los países de América Latina, las principales fuentes de información han sido estudios de caso, como Colombia (8), lo que los proyectos de reforma contemplan como posibles formas de contratación, como en Costa Rica (9) y, en los casos de Argentina y Chile, los estudios específicos sobre el tema publicados por la Comisión Económica para América Latina y el Caribe (CEPAL) (10-13).

En los últimos años, algunos países de la OCDE han experimentado reformas importantes que han provocado la separación de las funciones de financiación y compra de servicios de salud de las de producción, tradicionalmente desempeñadas de forma unificada por el sector público. Por ejemplo, en el

\section{La efectividad de la acción estatal y el conflicto político subyacente}

Uno de los ejes de la propuesta de reforma, la desregulación de la afiliación a las OS, se vio obstaculizada por la firme oposición de la Confederación General del Trabajo (CGT). Los líderes sindicales apelaron a argumentos de solidaridad para defender el mantenimiento de su control sobre recursos que constituyen una importante fuente de poder económico y político. El conflicto político e ideológico se centró en torno a las propuestas de organización elaboradas alternativamente por la CGT y el Banco Mundial, y a las alternativas de arbitraje y compromiso que intentaron construir entre ambas los funcionarios encargados de elaborar y poner en ejecución las decisiones del Estado.

\section{La propuesta de reforma respaldada por el Banco Mundial}

El crédito otorgado por el Banco Mundial a Argentina con el objeto de racionalizar la administración de las OS se aplicó a través del Programa de Apoyo a la Reforma del Sistema de Obras Sociales (PARSOS). Sus objetivos fueron sintetizados de la siguiente forma en una publicación del Ministerio de Trabajo:

- $\quad$ introducir competencia en el mercado financiador evitando la selección de riesgos;

- reasignar los recursos del Fondo Solidario de Redistribución ${ }^{4}$ sobre la base estricta del ingreso y los riesgos de los beneficiarios;

- desarrollar un marco regulador efectivo;

- desarrollar instituciones de seguro que promuevan la competencia, la transparencia y la rendición de cuentas para proteger los derechos de los consumidores;

- brindar asistencia técnica financiera a las OS y al Plan de Atención Médica Integral para Jubilados y Pensionados (PAMI), con el fin de mejorar su eficiencia y sus balances y de cumplir con las nuevas normas y regulaciones.

\footnotetext{
${ }^{4}$ El Fondo de Redistribución fue creado en 1970 por la ley 1.610, que sancionó la ampliación del sistema de OS para toda la población en relación de dependencia. La totalidad de las OS contribuía a este Fondo con un porcentaje de sus ingresos; luego los recursos así obtenidos debían canalizarse hacia las entidades con menor capacidad de cobertura, con el fin de atenuar las diferencias entre OS ricas y pobres. Sin embargo, la utilización política de los respectivos subsidios constituyó un mecanismo más de distorsión del sistema.
} 
Los funcionarios encargados de administrar el programa de reconversión de las OS financiado por el Banco Mundial propusieron también la definición de una canasta básica de servicios, destinada a garantizar un piso de cobertura igualitaria a todos los beneficiarios del sistema. En función del costo de ese programa se garantizaría un aporte mínimo a todos los beneficiarios titulares.

\section{La propuesta respaldada por la Confederación General del Trabajo}

Ante la amenaza planteada por la desregulación de las OS y la posibilidad de que posteriormente se abriera la competencia por los beneficiarios de la seguridad social a las empresas de medicina basadas en el pago por adelantado (prepago), la CGT adoptó una posición defensiva. Su propuesta consistió en modificaciones mínimas del status quo, conservando la estructura institucional existente y su autonomía, sin desarrollar competencia entre las OS ni someterse a las directivas del Banco Mundial, pero con el compromiso de introducir una estrategia para racionalizar el gasto. El programa de reconversión propuesto incluyó también la definición de un paquete básico de servicios, sancionado posteriormente como Programa Médico Obligatorio, y el otorgamiento de subsidios a aquellas OS que, debido a una insuficiencia de recursos, no estuvieran en condiciones de financiarlo. La propuesta también abarcaba el financiamiento del proceso de reorganización: racionalización del personal, renegociación de contratos con prestadores, adecuación de equipos e instalaciones y cancelación de pasivos corrientes mediante préstamos a 48 meses con 18 meses de gracia.

\section{La política de compromiso llevada adelante por el gobierno}

Dos decretos sancionados por el poder ejecutivo en 1995 se encaminaron a conciliar las posiciones en conflicto. Un número relativamente importante de OS aceptó someterse a las directivas del Banco Mundial, tendientes a sanear su administración, eliminar el personal administrativo excedente, racionalizar el gasto y alentar la fusión entre OS con una cantidad de beneficiarios insuficiente para garantizar un adecuado reparto de riesgos. Los resultados parecieron prometedores en el corto plazo. Sin embargo, la estructura general del sistema siguió presentando distorsiones que obstaculizaban el cumplimiento adecuado de su función de cobertura, mientras la solidaridad proclamada como pilar del modelo de organización pugnaba con la creciente estratificación de la población cubierta.
Reino Unido las autoridades sanitarias locales y los GP Fundholders compran los servicios de salud a los trusts hospitalarios del Sistema Nacional de Salud (National Health System: NHS), a los hospitales privados y a los servicios comunitarios. En Finlandia y Suecia son los municipios y los condados, respectivamente, quienes realizan la función de contratación y compra de la asistencia médica a los ciudadanos. La separación de la compra y la producción de servicios de salud ha permitido definir mejor las funciones y responsabilidades sanitarias, ha orientado los incentivos de los agentes hacia el control de costos y, en ese sentido, ha provocado modificaciones en las formas de pago a los prestadores.

Elementos similares se observan en Colombia con la separación de las Entidades Promotoras de Salud (EPS) y las Instituciones Prestadoras de Salud (IPS), y en Argentina, donde la mayoría de las Obras Sociales contratan la prestación de los servicios con prestadores privados.

Uno de los aspectos que llama la atención en los países de América Latina es la coexistencia de formas diversas de pago a los prestadores, debidas en parte a los procesos de reforma en que se hallan inmersos y al peso nada despreciable que el sector privado tiene en sus sistemas de salud (cuadro 3). El estudio de los países de la OCDE pone de manifiesto algo sobre lo que ya se había hecho hincapié anteriormente: la inexistencia de un método de contratación óptimo que cumpla todos los objetivos de política sanitaria, así como la relevancia de la organización de los sistemas sanitarios en la elección del mismo (cuadro 2).

Si nos centramos en las formas de pago en la atención primaria, podemos distinguir dos tendencias claras. En primer lugar, se observa una orientación hacia los sistemas mixtos de remuneración a los médicos, con un componente prospectivo importante. Las formas de pago se combinan en estos modelos provocando un "tira y afloja" entre incentivos contrapuestos. En bastantes países de la OCDE, el componente prospectivo de la remuneración de los 
médicos de atención primaria está basado en un pago capitativo, combinado bien con pagos por acto médico, (por ejemplo, en Dinamarca o en el "modelo Estocolmo" de Suecia), bien con pagos por objetivos logrados (por ejemplo, en el Reino Unido), o bien con remuneraciones salariales (por ejemplo, en España y en Finlandia).

El pago por capitación tiene un protagonismo importante en los países de América Latina analizados. En estos casos se utiliza también para garantizar un conjunto mínimo de prestaciones a segmentos marginados de la población, como sucede en Colombia. La tendencia de estos países es que esta forma de pago sea un componente fijo, pero no exclusivo, en la remuneración de la atención ambulatoria.

En segundo lugar, destaca cómo los países que remuneran a sus médicos por servicio tratan de limitar la discrecionalidad que esta forma de pago incentiva, buscando un equilibrio entre la calidad de los servicios prestados y la eficiencia en la utilización de los recursos. Este empeño se materializa, por una parte, en la aplicación de sistemas de tarifas prospectivas en los casos de Alemania, Francia y del Medicare de los Estados Unidos; por otra parte, en la cesión de responsabilidades a las asociaciones de médicos, que están capacitadas no solo para distribuir el presupuesto inicial asignado, sino también para penalizar a sus miembros en caso de sobreutilización, como en Alemania, $y$, por último, en el establecimiento de límites al gasto global, como sucede en algunas provincias de Canadá.

En cuanto a la atención hospitalaria, se está experimentando un giro desde los presupuestos globales basados en costos históricos hacia modelos de contratación prospectivos por costo y volumen, o costo por caso. La remuneración basada en GDR o similares, se lleva a cabo en Alemania, Estados Unidos, Francia y Suecia, entre otros, y también se está ensayando en Chile y Colombia. Sin embargo, tanto en el caso de los GDR como de las unidades ponderadas asistenciales en España o las unidades de producción hospitalaria en Costa Rica, su implementación está limitada por las grandes inversiones

\section{Los cambios en las relaciones entre obras sociales y prestadores de servicios}

Las estrategias puestas en marcha por financiadoras y prestadores para superar la crisis financiera del sistema se han traducido en transformaciones institucionales, cambios en las formas de contratación entre la oferta y la demanda de servicios de salud, cambios en la organización y el control de los procesos de trabajo, y la introducción de nuevas modalidades de remuneración a los prestadores de servicios de salud.

El conflicto se instaló en el seno de las corporaciones profesionales cuando las instituciones con mayor capacidad de controlar sus costos abandonaron el paraguas protector del oligopolio corporativo y entraron en franca competencia con sus pares por la captación de los contratos con las OS. Las nuevas modalidades de contrato -identificadas como contratos de riesgo- abandonaron la práctica generalizada del pago por servicio y se basaron en el compromiso de brindar servicios integrales a una población determinada a partir de un pago per cápita.

Los cambios en la organización de la oferta suscitados por esta nueva modalidad de contratos incluyen el surgimiento de nuevas empresas y nuevas formas de vinculación entre prestadores. En primer lugar, surge la organización de empresas Administradoras o Gerenciadoras de Prestaciones, las cuales operan bajo dos modalidades fundamentales: a) negociando con las entidades financiadoras los nuevos contratos a base de un pago per cápita y subcontratando la provisión de servicios con prestadores directos; b) haciéndose cargo de la gestión de los contratos formalizados entre OS y prestadores directos. En segundo lugar, se multiplican las modalidades de organización de los prestadores, ya sea para contratar directamente con las OS o para hacerse cargo de la provisión de servicios a las empresas u organizaciones titulares de contratos de riesgo. Se produce así la conformación de Uniones Transitorias de Empresas (UTE), Redes o Asociaciones de Prestadores encargadas de la provisión directa de los servicios.

La nueva modalidad de pago por las OS - una suma mensual preestablecida por beneficiario destinada a la entidad responsable de su atención médica- supone que los prestadores asumen el riesgo financiero inherente a la prestación de servicios a la población cubierta. La remuneración per cápita tiene, por consiguiente, un doble efecto: permite a las OS administrar mejor sus recursos, descargando el riesgo propio de la sobreutilización, sobreprestación y sobrefacturación en las Redes, UTE, o Administradoras de Prestaciones. Al mismo tiempo, 
el mecanismo de pago per cápita cambia las expectativas de los prestadores en cuanto a sus beneficios: ya no se trata de aumentar el ingreso a partir de la expansión de la capacidad instalada, o de la cantidad y complejidad de los servicios provistos, sino de administrar la atención de manera que no se supere el techo impuesto al gasto total por la existencia de un monto de recursos financieros predeterminado.

Hay una complejidad adicional, sin embargo, en la forma en que se estructura el mercado en Argentina: la remuneración de los prestadores directos sigue realizándose sobre la base del honorario por servicio, o de días-cama, aunque se incluyen también formas como el pago por módulo, utilizado para la retribución de determinadas prácticas de internación. Por esta razón, los términos del acuerdo entre la empresa que detenta el contrato con la entidad financiadora y los prestadores directos, en lo relativo a la asignación del riesgo cuando la retribución de los servicios prestados supere el monto percibido per cápita, determinarán en muchos casos la viabilidad financiera del convenio.

\section{Conclusiones}

Los cambios producidos en la operación del sistema no pueden atribuirse en forma directa a la voluntad de los reformadores. Las estrategias de los nuevos y viejos actores con protagonismo en el sector de la salud, quienes operan dentro de un sistema fragmentado, han tenido como consecuencia el crecimiento de la heterogeneidad preexistente. Esta heterogeneidad, al igual que en el modelo anterior, significa una asignación deficiente de los recursos disponibles y una mayor brecha en el acceso a la atención médica entre distintos sectores de beneficiarios, situación que acentúa aun más la falta de equidad. Los objetivos de lograr que los beneficiarios en la base del sistema gocen de igualdad, proteger a la población de menores recursos, dejar librada a la decisión individual la contratación de diferentes planes de seguro y estimular la reducción de los costos y el incremento de la eficiencia asignativa y productiva a partir de la competencia entre sus agentes, no han sido alcanzados.

La política de limitar las funciones del Estado y de confiar en las reglas del mercado para desalentar las estrategias individuales y corporativas que obstaculizaban el cumplimiento de los objetivos sociales asignados al sistema también se ha mostrado infructífera. El sistema de servicios de atención médica tampoco ha servido como mecanismo de compensación de que requiere el sistema de información necesario para generar los datos exigidos para desarrollar este tipo de sistemas de pago.

Siguiendo el marco teórico expuesto en la sección correspondiente, el cuadro 4 presenta la gama de posibles sistemas de remuneración que se derivan de la combinación de los dos vectores: el correspondiente a los incentivos y el relativo a la distribución de riesgos. En el eje vertical están colocados, de menor a mayor agregación, las unidades de pago que generan los incentivos en términos de eficiencia técnica. En el eje horizontal están representadas posibles distribuciones de riesgos financieros. En el extremo izquierdo se hallan los sistemas que asignan mayor riesgo a los prestadores, al incorporar un menor ajuste a los sistemas de pago, y en el extremo derecho los que atribuyen mayor riesgo al financiador, con un mayor refinamiento de los sistemas de pago. En este cuadro hemos situado algunos de los sistemas de pago propios de algunos países de la OCDE y de América Latina que acabamos de analizar.

\section{CONCLUSIONES}

El sistema de pago a prestadores define una parte fundamental de los incentivos contractuales entre los agentes de los sistemas de salud y es uno de los signos característicos de estos. Como en cualquier forma contractual, cada una de las formas de pago tiene aspectos positivos y negativos que se deben evaluar en función de los objetivos que se pretenden alcanzar.

A pesar de la complejidad de los sistemas de pago, en este artículo se ha ofrecido un marco conceptual sistemático para su análisis. Este marco se basa en la descripción de los sistemas de pago en función de dos dimensiones: la unidad de pago que utilizan y la distribución de riesgos financieros entre el financiador y el prestador de la atención sanitaria. Estos dos aspectos definen las coordenadas de un amplio espectro de posibles formas de pago. En un extremo tenemos la remuneración fija, o sea, con un solo inter- 
las desigualdades producidas en el aparato productivo y la distribución del ingreso. La estratificación que en él se opera configura un mecanismo de exclusión adicional, lo cual es consecuencia de la crisis financiera del sistema y de las estrategias puestas en marcha para superarla.

Los retos que sigue habiendo en la actualidad son básicamente los siguientes:

- $\quad$ Existen dos mundos muy diferenciados: el del sector poblacional que tiene acceso a prácticas médicas de alta complejidad, sean o no necesarias, y el del sector social excluido, que carece de acceso a la atención médica básica para garantizar su derecho a la salud. La reforma efectuada no ha logrado superar esta situación.

- Dado que actualmente la mitad de la población argentina carece de cobertura de salud por dependencia, la cual es producto del desempleo y de la inestabilidad laboral de los últimos lustros, el proceso de reforma descrito operó sobre la población menos desfavorecida. La población con más necesidades sigue contando solamente con los servicios del sector público, que en muchos lugares, sobre todo en los sitios alejados de las grandes ciudades, son muy deficientes.

- El debate centrado en el establecimiento de un techo de financiación por beneficiario y en el ajuste de las prestaciones brindadas bajo dicho límite, dificultó la discusión profunda de la transformación del modelo de atención para garantizar cobertura universal con equidad a toda la población, independientemente de su inserción en el mercado laboral.

- $\quad$ La red pública de prestadores corre el riesgo de quedar encuadrada como un prestador de servicios más, en competencia con el resto, quedando desvirtuado su potencial como compensador de desigualdades y creador de nuevos conceptos y prácticas de salud y de atención al enfermo.

- La eventual evolución del proceso de desregulación hacia la conformación de un oligopolio de grandes entidades financieras dentro del sector de la salud, pone en duda la capacidad real de regulación con la que contará el Estado en ese contexto. valo. Es el pago prospectivo más puro, o sin ajustes, como, por ejemplo, el pago capitativo. En este caso, los prestadores soportan todo el riesgo financiero derivado de la variabilidad de costos para una tarifa prefijada y una determinada unidad de pago. En el otro extremo tenemos una infinidad de intervalos. Es el caso del pago retrospectivo o pago por acto. El riesgo incurrido por los prestadores es nulo: sea cual sea el costo de la unidad producida, la remuneración lo cubrirá y el financiador será quien soporte todo el riesgo financiero derivado de la variabilidad de costos.

Como suele ocurrir, los extremos no parecen óptimos. De modo que la cuestión consiste en escoger un sistema de remuneración intermedio, eligiéndolo de entre una gama de posibles sistemas. Para ello, es necesario seleccionar, por una parte, la unidad de pago que consideremos óptima según los objetivos del financiador $y$, por otra parte, una distribución de riesgos que atribuya al prestador los riesgos derivados del mayor o menor grado de eficiencia que alcance en el suministro de los servicios.

El problema es que se desconoce dónde está la frontera que separa las variaciones injustificables, debidas a ineficiencias, de las justificables. Estas últimas están fuertemente relacionadas con la heterogeneidad del producto sanitario y sus causas quedan generalmente fuera del control del prestador.

Para resolver el dilema de la elección entre los pagos prospectivos, con dificultades de distribución de riesgos, y los retrospectivos, que suelen proporcionar incentivos al despilfarro de recursos y a la ineficiencia, los sistemas mixtos son los que muestran más ventajas, aunque no resuelven el problema de la selección de la unidad de pago.

En este artículo hemos presentado un resumen de las formas de pago vigentes en 10 países de la OCDE y cuatro países de América Latina, analizados desde la perspectiva del modelo expuesto. De la visión global de todos ellos se extraen algunas conclusiones.

Por una parte, observamos que la remuneración de la atención ambulatoria sigue dos tendencias claras. En pri- 
CUADRO 4. Visión bidimensional de los sistemas de financiación

\begin{tabular}{|c|c|c|c|c|c|c|c|}
\hline \multirow{2}{*}{$\begin{array}{c}\begin{array}{c}\text { Amplitud de } \\
\text { incentivos }\end{array} \\
\text { Limitados }\end{array}$} & \multirow{2}{*}{$\begin{array}{l}\begin{array}{c}\text { Unidad de } \\
\text { pago }\end{array} \\
\text { Servicio }\end{array}$} & \multicolumn{6}{|c|}{ Riesgos: $100 \%$ Prestador...... } \\
\hline & & & & M ISAPRE Chile & M Alemania & M Canadá & M Francia \\
\hline & Ingreso & & & & $\begin{array}{l}\text { H Suecia } \\
\text { GDR }\end{array}$ & & PAD Chile \\
\hline & Capitación & $\begin{array}{l}\text { "GP fundholders" } \\
\text { MUFACE España } \\
\text { M Estocolmo }\end{array}$ & \multicolumn{2}{|c|}{$\begin{array}{l}\text { MA España } \\
\text { M Medicare }\end{array}$} & anda & & \\
\hline
\end{tabular}

Amplios $\quad$ Presupuesto global H Holanda H Canadá H Francia

M: médicos; H: hospitales; MA: médicos de atención ambulatoria; MH: médicos de atención hospitalaria; PAD: Pagos Asociados a Diagnóstico; GDR: Grupos de Diagnóstico Relacionados; MUFACE: Mutualidad de Funcionarios de la Adminsitración Central.

mer lugar, muestra una orientación hacia los sistemas mixtos de remuneración a los médicos, con un componente prospectivo importante. De este modo, las formas de pago se combinan provocando una tensión entre incentivos contrapuestos.
En segundo lugar, los países que remuneran a sus médicos por servicio tratan de limitar la discrecionalidad que incentiva esta forma de pago buscando un equilibrio entre la calidad de los servicios prestados y la eficiencia en la utilización de los recursos.

\section{REFERENCIAS}

1. Pellisé L. Sistemas de pago óptimos en el sector sanitario. En: Del Llano J, Ortún V, Martín JM, Millán J, Gené J, eds. Gestión sanitaria. Innovaciones y desafíos. Barcelona: Masson, BCN; 1998.

2. Newhouse JP. Cream-skimming, asymmetric information and a competitive insurance market. J Health Econ 1984;3:97-100.

3. Álvarez B, Lobo F, Pellisé L. Sistemas de pago a proveedores de servicios sanitarios en países latinoamericanos y de la OCDE. Washington, D.C.: División de Desarrollo de Sistemas y Servicios de Salud. Organización Panamericana de la Salud; 1998. (Serie Organización y Gestión de Sistemas y Servicios de Salud, 12).

4. Organization for Economic Cooperation and Development. The reform of health care. A comparative analysis of seven OECD countries. Paris: OECD; 1992.

5. Organization for Economic Cooperation and Development. The reform of health care. A comparative analysis of seven OECD countries. Paris: OECD; 1994.
6. Saltman RB, Figueras J. Reforma sanitaria en Europa. Análisis de las estrategias actuales. Madrid: Ministerio de Sanidad y Consumo y Organización Mundial de la Salud; 1997.

7. Schneider M, Biene-Dietrich P, Gabanyi M, et al. Gesundheitssysteme im internationalen Vergleich. Ausgabe 1994. Laufende Berichterstattung zu ausländischen Gesundheitssystemen, Basys. Ausburg; 1995.

8. Gutiérrez C, Molina CG, Wüllner A. Las formas de contratación entre prestadoras y administradoras de salud. Sus perspectivas en el nuevo marco de la Seguridad Social. Santa Fe de Bogotá: Fundación Corona, Fundación para la Educación Superior y Fundación Restrepo Barco; 1996

9. Andersen Consulting y Consorci Hospitalari de Catalunya. Diseño e implantación de mecanismos de asignación de recursos y para la modernización de los procesos críti-cos de las gerencias financiera y médica. San José: Caja Costarricense de Seguro Social; 1996
Por último, comprobamos que la atención hospitalaria está experimentando un giro desde los presupuestos globales basados en costes históricos hacia los modelos de contratación prospectivos definidos por coste y volumen o coste por caso.
10. Aedo C. La competencia manejada y reformas para el sector salud de Chile. Serie Financiamiento del Desarrollo, 50. Santiago de Chile: Comisión Económica para América Latina y el Caribe, Naciones Unidas; 1997.

11. Bitrán R, Almarza FX. Las Instituciones de Salud Previsional (ISAPRE) en Chile. Serie de Financiamiento del Desarrollo, 56. Santiago de Chile: Comisión Económica para América Latina y el Caribe, Naciones Unidas; 1997.

12. Montoya S. Reformas al financiamiento del Sistema de Salud en Argentina. Santiago de Chile: Comisión Económica para América Latina y el Caribe, Naciones Unidas; 1997. (Serie Financiamiento del Desarrollo, 60).

13. Tafani R. Reforma al Sector Salud en Argentina. Serie Financiamiento del Desarrollo, 53. Santiago de Chile: Comisión Económica para América Latina y el Caribe, Naciones Unidas; 1997. 
ABSTRACT The system used to pay health services providers is one of the most important components of the contractual relationship between persons who receive health services and the individual practitioners and institutions that provide those services. That

Payment systems for health services providers in countries of Latin America and of the OECD payment system is also relevant in assessing a health system, including its efficiency and quality. In this article we present a simple analytical framework for various payment systems. We also provide an overview of the payment approaches used in two groups of countries whose experiences we consider representative: 10 nations of the Organization for Economic Cooperation and Development (OECD) and four countries of Latin America. We present a basic model to characterize the different forms of payment based on two dimensions. One of the dimensions is the payment "unit," which is used to measure the amount of health care services provided or promised. The other dimension is the distribution of financial risks between the service provider and the service purchaser. Each payment system has advantages and disadvantages that should be evaluated in relation to the intended objectives. On one extreme of the approaches is fixed remuneration, without any adjustments; it represents the purest prepayment approach. One example of fixed remuneration is capitated payment, in which providers carry all the financial risks coming from the variability in the cost of providing services. On the other extreme is fee-for-service payment, where service providers are not at financial risk; the insurer or other financing institution carries all the risk from variable costs. Neither of the extremes appears to be the best choice, and so the issue becomes one of selecting a remuneration system that falls between those extremes. Therefore, it is necessary to choose, on the one hand, the optimal payment unit according to the objectives of the financing entity and, on the other hand, a risk distribution approach that allocates to the service provider the risks coming from greater or less efficiency in delivering services. 